\title{
CONTINUED FRACTIONS FOR SQUARE SERIES GENERATING FUNCTIONS
}

\author{
MAXIE D. SCHMIDT \\ MAXIEDS@GMAIL.COM
}

\begin{abstract}
We consider new series expansions for variants of the so-termed ordinary geometric square series generating functions originally defined in the recent article titled "Square Series Generating Function Transformations" (arXiv: 1609.02803). Whereas the original square series transformations article adapts known generating function transformations to construct integral representations for these square series functions enumerating the square powers of $q^{n^{2}}$ for some fixed non-zero $q$ with $|q|<1$, we study the expansions of these special series through power series generated by Jacobi-type continued fractions, or J-fractions. We prove new exact expansions of the $h^{t h}$ convergents to these continued fraction series and show that the limiting case of these convergent generating functions exists as $h \rightarrow \infty$.

We also prove new infinite $q$-series representations of special square series expansions involving squarepower terms of the series parameter $q$, the $q$-Pochhammer symbol, and double sums over the $q$-binomial coefficients. Applications of the new results we prove within the article include new $q$-series representations for the ordinary generating functions of the special sequences, $r_{p}(n)$, and $\sigma_{1}(n)$, as well as parallels to the examples of the new integral representations for theta functions, series expansions of infinite products and partition function generating functions, and related unilateral special function series cited in the first square series transformations article.
\end{abstract}

Keywords: square series; q-series; J-fraction; continued fraction; sum of squares functions; sum of divisors function; theta function; ordinary generating function.

MSC Subject Codes: 05A15; 11 Y65; 11B65; $40 A 15$.

\section{INTRODUCTION}

1.1. Continued Fraction Expansions of Ordinary Generating Functions. Jacobi-type continued fractions, or J-fractions, correspond to power series defined by infinite continued fraction expansions of the form

$$
\begin{aligned}
J_{\infty}\left(\left\{c_{i}\right\},\left\{\mathrm{ab}_{i}\right\} ; z\right)= & \frac{1}{1-c_{1} z-\frac{\mathrm{ab}_{2} z^{2}}{1-c_{2} z-\frac{\mathrm{ab}_{3} z^{2}}{\cdots}}} \\
& =1+c_{1} z+\left(\mathrm{ab}_{2}+c_{1}^{2}\right) z^{2}+\left(2 \mathrm{ab}_{2} c_{1}+c_{1}^{3}+\mathrm{ab}_{2} c_{2}\right) z^{3}+\cdots,
\end{aligned}
$$

for some sequences $\left\{c_{i}\right\}_{i=1}^{\infty}$ and $\left\{\mathrm{ab}_{i}\right\}_{i=2}^{\infty}$, and some (typically formal) series variable $z \in \mathbb{C}[5, c f . \S 3.10]$ [8]. The formal series enumerated by special cases of the truncated and infinite J-fraction series of this form include ordinary (as opposed to typically closed-form exponential) generating functions for many one and two-index combinatorial sequences including the generalized factorial functions studied in the references and in the results from Flajolet's articles. The references provide infinite formal continued fraction series for the typically divergent ordinary generating functions of many special sequences, factorial functions, and combinatorial triangles arising in applications $[1,2,4,6]$.

1.2. J-Fraction Expansions of Ordinary Square Series Generating Functions. In this article, we consider a $q$-series-related variant of the J-fractions in (1) enumerating the square-power terms of $q^{n^{2}}$, which we have discovered computationally (see [7] for integral representations). In particular, for non-zero

Date: Revised 2017.02.17-v2. 
$q, z \in \mathbb{C}$ such that $|q|,|z|<1$, we define the following Jacobi-type J-fraction, $J_{\infty}^{[\mathrm{sq}]}(q, z)$, for the ordinary square series generating function defined in [7] as follows:

$$
J_{\infty}^{[\mathrm{sq}]}(q, z)=\frac{1}{1-q z-\frac{q^{2}\left(q^{2}-1\right) z^{2}}{1-q\left(q^{4}+q^{2}-1\right) z-\frac{q^{8}\left(q^{4}-1\right) z^{2}}{1-q^{3}\left(q^{6}+q^{4}-1\right) z-\frac{q^{14}\left(q^{6}-1\right) z^{2}}{\cdots}}}}
$$

Definition 1.1 (Component Sequences and Convergent Functions). We define the component sequence functions, $c_{h} \equiv c_{h}^{[\mathrm{sq}]}(q)$ and $\mathrm{ab}_{h} \equiv \mathrm{ab}_{h}^{[\mathrm{sq}]}(q)$, as follows for $h \geq 0$ :

$$
\begin{array}{r}
c_{h}^{[\mathrm{sq}]}(q):= \begin{cases}q^{2 h-3} \cdot\left(q^{2 h}+q^{2 h-2}-1\right) & \text { if } h \geq 2 ; \\
q & \text { if } h=1 ; \\
1 & \text { if } h=0\end{cases} \\
\operatorname{ab}_{h}^{[\mathrm{sq}]}(q):= \begin{cases}q^{6 h-10} \cdot\left(q^{2 h-2}-1\right) & \text { if } h \geq 2 ; \\
0 & \text { otherwise. }\end{cases}
\end{array}
$$

We then define the $h^{\text {th }}$ convergent functions, $\operatorname{Conv}_{h}(q, z):=\mathrm{P}_{h}(q, z) / \mathrm{Q}_{h}(q, z)$, recursively through the component numerator and denominator functions given by ${ }^{1}$

$$
\begin{aligned}
& \mathrm{P}_{h}(q, z)=\left(1-c_{h}^{[\mathrm{sq}]}(q) \cdot z\right) \mathrm{P}_{h-1}(q, z)-\mathrm{ab}_{h}^{[\mathrm{sq}]}(q) \cdot z^{2} \mathrm{P}_{h-2}(q, z)+[h=1]_{\delta} \\
& \mathrm{Q}_{h}(q, z)=\left(1-c_{h}^{[\mathrm{sq}]}(q) \cdot z\right) \mathrm{Q}_{h-1}(q, z)-\mathrm{ab}_{h}^{[\mathrm{sq}]}(q) \cdot z^{2} \mathrm{Q}_{h-2}(q, z)+\left(1-c_{1}^{[\mathrm{sq}]}(q) \cdot z\right)[h=1]_{\delta}+[h=0]_{\delta} .
\end{aligned}
$$

We claim, and subsequently prove in Section 2, that the truncated $(h+1)$-order power series expansions of the $h^{\text {th }}$ convergent functions, $\operatorname{Conv}_{h}(q, z)$, defined in Definition 1.1 generate the so-termed "square series" powers defined in [7] as

$$
\begin{aligned}
& \operatorname{Conv}_{h}(q, z)=\frac{1}{1-q z-\frac{q^{2}\left(q^{2}-1\right) z^{2}}{1-q\left(q^{4}+q^{2}-1\right) z-\frac{q^{8}\left(q^{4}-1\right) z^{2}}{1-q^{3}\left(q^{6}+q^{4}-1\right) z-\frac{q^{14}\left(q^{6}-1\right) z^{2}}{\cdots}}}} \\
& =1+q z+q^{4} z^{2}+q^{9} z^{3}+\cdots+q^{h^{2}} z^{h}+\sum_{n=h+1}^{\infty} \widetilde{e}_{h, n}(q) \cdot z^{n},
\end{aligned}
$$

for all $n, h \in \mathbb{Z}^{+}$. More precisely, we state the next result as our main theorem which we carefully prove in Section 2.2 of the article below.

Theorem 1.2 (J-Fraction Expansions of Ordinary Square Series Generating Functions). For all $h \geq 1$ and every $0 \leq n \leq h$, we have that $\left[z^{n}\right] \operatorname{Conv}_{h}(q, z)=q^{n^{2}}$.

For $0<|q|,|z|<1$, we show that the limiting convergent function, denoted by

$$
J_{\infty}^{[\mathrm{sq}]}(q, z):=\sum_{n \geq 0} q^{n^{2}} z^{n}=\lim _{h \longrightarrow \infty} \operatorname{Conv}_{h}(q, z), \quad \text { (Ordinary Geometric Square Series) }
$$

\footnotetext{
${ }^{1}$ Notation: Iverson's convention compactly specifies boolean-valued conditions and is equivalent to the Kronecker delta function, $\delta_{i, j}$, as $[n=k]_{\delta} \equiv \delta_{n, k}$. Similarly, [cond $=$ True $]_{\delta} \equiv \delta_{\text {cond, True }}$ in the remainder of the article.
} 
which corresponds to the integral representations of the ordinary geometric square series cases defined in [7] given by

$$
J_{\infty}^{[\mathrm{sq}]}(q, z)=\int_{0}^{\infty} \frac{2 e^{-t^{2} / 2}}{\sqrt{2 \pi}}\left[\frac{1-z \cosh (t \sqrt{2 \log (q)})}{z^{2}-2 z \cosh (t \sqrt{2 \log (q)})+1}\right] d t
$$

(Ordinary Geometric Square Series Integral Representation)

exists, and that the limiting cases of this convergent function series converge uniformly as a function of $z$ with respect to the sequence of $h^{\text {th }}$ partial sums of (6) defined below.

Thus we are able to prove new infinite $q$-series identities generating special cases of $J_{\infty}^{[\mathrm{sq}]}\left(q, c \cdot q^{p}\right)$ in Section 2, which arise in many applications to theta functions and special function series. The primary new form of the infinite q-series expansions we prove in Section 2 of the article has the form

$$
J_{\infty}^{[\mathrm{sq}]}(q, z)=\sum_{i=1}^{\infty} \frac{(-1)^{i-1} q^{(3 i-4)(i-1)}\left(q^{2} ; q^{2}\right)_{i-1} z^{2 i-2}}{\sum_{0 \leq j \leq n<2 i}\left[\begin{array}{l}
i \\
j
\end{array}\right]_{q^{2}}\left[\begin{array}{l}
i-1 \\
n-j
\end{array}\right]_{q^{2}} q^{2 j} \cdot\left(-q^{2 i-3} \cdot z\right)^{n}} .
$$

Moreover, the $h^{\text {th }}$ partial sums of the infinite $q$-series expansion in (6) corresponding to the $h^{\text {th }}$ convergent function, $\operatorname{Conv}_{h}(q, z)$, is always at least $(h+1)$-order accurate in generating the coefficients, $q^{n^{2}}$, of $z^{n}$ for $0 \leq n \leq h$. In addition, we show that we can differentiate these new infinite square series expansions termwise with respect to $z$ thus allowing us to generate series for higher-order derivatives of the Jacobi theta functions, which are generated as particular cases of the geometric square series, $\vartheta_{0, m}(a, b ; q, z):=$ $\sum_{n}(a n+b)^{m} q^{n^{2}} z^{n}$, in [7] and which similarly arise in many well-known identities and classical expansions of special products and partition function generating functions.

\begin{tabular}{|l|l|}
\hline$h$ & $\mathrm{P}_{h}(q, z)$ \\
\hline 1 & 1 \\
2 & $1-q\left(q^{4}+q^{2}-1\right) z$ \\
3 & $1-q\left(1+q^{2}\right)\left(-1+q^{2}+q^{6}\right) z+q^{4}\left(1-q^{2}-q^{4}+q^{8}+q^{10}\right) z^{2}$ \\
4 & $1-q\left(1-q+q^{2}\right)\left(1+q+q^{2}\right)\left(-1+q^{2}+q^{8}\right) z+q^{4}\left(1-q^{4}-q^{6}-q^{8}+q^{12}+2 q^{14}+q^{16}+q^{18}\right) z^{2}$ \\
& $\quad-q^{9}\left(-1+q^{2}+q^{4}-2 q^{10}+q^{16}+q^{18}\right) z^{3}$ \\
5 & $1-q\left(1+q^{2}\right)\left(1+q^{4}\right)\left(1-q^{2}+q^{4}\right)\left(-1+q^{4}+q^{6}\right) z$ \\
& $\quad+q^{4}\left(1+q^{2}\right)\left(1-q+q^{2}\right)\left(1+q+q^{2}\right)\left(1-q^{2}+q^{4}\right)\left(1-q^{2}-q^{6}+q^{12}+q^{16}\right) z^{2}$ \\
& $-q^{9}\left(1+q^{2}\right)\left(-1+q^{2}+q^{6}-2 q^{14}-q^{18}+q^{20}+2 q^{24}+q^{28}\right) z^{3}$ \\
& $+q^{16}\left(1-q^{2}-q^{4}+q^{10}+q^{12}+q^{14}-q^{16}-q^{18}-q^{20}+q^{26}+q^{28}\right) z^{4}$ \\
\hline
\end{tabular}

TABlE 1. The Numerator Convergent Functions, $\mathrm{P}_{h}(q, z)$

Tables of the expansions of the component convergent functions, $\mathrm{P}_{h}(q, z)$ and $\mathrm{Q}_{h}(q, z)$, for the first few special cases of $h \geq 1$ are given in Table 1 and Table 2. Proposition 2.2 and Proposition 2.4 stated in Section 2 of the article below provide more general exact formulas satisfied by these convergent function sequences for all $h \geq 1$. Since the sums of squares functions, $r_{p}(n)$, are generated by the $p^{\text {th }}$ powers of the Jacobi theta function, $\vartheta_{3}(q)=1+2 q+2 q^{4}+2 q^{9}+\cdots$, we are particularly interested in the properties of the convergent function special cases, $\widetilde{\vartheta}_{3, h}(q):=1+2 q \cdot \operatorname{Conv}_{h}\left(q, q^{2}\right)$, in the context of the applications briefly motivated in the next subsection (see Table 3 on page 13).

1.3. Generating the Sums of Squares Functions. In Section 3, we use the new square series J-fraction representation for $\widetilde{\vartheta}_{3, h}(q)$, which we will prove in the next section, to expand new forms of congruences and generating functions for the sums of squares functions, $r_{2}(n)$, and more generally for $r_{p}(n)$ when $p \geq 3$. For a fixed integer $p \geq 2$, the function, $r_{p}(n)$, is defined to be the number of solutions to the equation

$$
n=x_{1}^{2}+x_{2}^{2}+\cdots+x_{p}^{2},
$$




\begin{tabular}{|c|c|}
\hline$h$ & $\overline{\mathrm{Q}_{h}(q, z)}$ \\
\hline 0 & 1 \\
\hline 1 & $1-q z$ \\
\hline 2 & $1-q^{3}\left(1+q^{2}\right) z+q^{6} z^{2}$ \\
\hline 3 & $1-q^{5}\left(1-q+q^{2}\right)\left(1+q+q^{2}\right) z+q^{10}\left(1-q+q^{2}\right)\left(1+q+q^{2}\right) z^{2}-q^{15} z^{3}$ \\
\hline 4 & $\begin{array}{l}1-q^{7}\left(1+q^{2}\right)\left(1+q^{4}\right) z+q^{14}\left(1-q+q^{2}\right)\left(1+q+q^{2}\right)\left(1+q^{4}\right) z^{2} \\
\quad-q^{21}\left(1+q^{2}\right)\left(1+q^{4}\right) z^{3}+q^{28} z^{4}\end{array}$ \\
\hline 5 & $\begin{array}{l}1-q^{9}\left(1-q+q^{2}-q^{3}+q^{4}\right)\left(1+q+q^{2}+q^{3}+q^{4}\right) z \\
\quad+q^{18}\left(1+q^{4}\right)\left(1-q+q^{2}-q^{3}+q^{4}\right)\left(1+q+q^{2}+q^{3}+q^{4}\right) z^{2} \\
\quad-q^{27}\left(1+q^{4}\right)\left(1-q+q^{2}-q^{3}+q^{4}\right)\left(1+q+q^{2}+q^{3}+q^{4}\right) z^{3} \\
\quad+q^{36}\left(1-q+q^{2}-q^{3}+q^{4}\right)\left(1+q+q^{2}+q^{3}+q^{4}\right) z^{4}-q^{45} z^{5}\end{array}$ \\
\hline
\end{tabular}

TABle 2. The Denominator Convergent Functions, $\mathrm{Q}_{h}(q, z)$

where the integers $x_{i}$ are taken to be positive, negative, or zero-valued. For each $p \geq 2$, these sequences are generated over $q$ by the following $p^{\text {th }}$ powers of the Jacobi theta function, $\vartheta_{3}(q)[5,3]$ :

$$
\sum_{n \geq 0} r_{p}(n) q^{n}=\vartheta_{3}(q)^{p}=\left(1+2 q \times \sum_{n=0}^{\infty} q^{n(n+2)}\right)^{p} .
$$

If we let $\operatorname{Conv}_{h}(q, z):=P_{h}(q, z) / Q_{h}(q, z)$ denote the $h^{\text {th }}$ convergent function to our new J-fraction expansion, as in Definition 1.1, and let $E_{h}\left(z^{m}\right):=z^{m} \cdot[0 \leq m \leq h]_{\delta}$ denote the erasing operator, or $(h+1)$-order power series truncation operator in $z$, we can generate the sums of squares functions by

$$
\sum_{0 \leq n \leq h} q^{n^{2}}\left(q^{2} z\right)^{n}=E_{h}\left[\left(\frac{Q_{h}\left(q, q^{2} z\right)+2 q P_{h}\left(q, q^{2} z\right)}{Q_{h}\left(q, q^{2} z\right)}\right)^{p}\right] .
$$

In Section 3.1, we give a new explicit formula for the generating functions of $r_{p}(n)$ for all $p \geq 2$ in the form of

$$
r_{p}(n)=\left[q^{n}\right]\left(1+2 q \times \sum_{i=1}^{\infty} \frac{(-1)^{i-1} q^{3 i(i-1)}\left(q^{2} ; q^{2}\right)_{i-1}}{\sum_{0 \leq n<2 i}\left(\sum_{0 \leq j \leq n}\left[\begin{array}{l}
i \\
j
\end{array}\right]_{q^{2}}\left[\begin{array}{c}
i-1 \\
n-j
\end{array}\right]_{q^{2}} q^{2 j}\right)\left(-q^{2 i-1}\right)^{n}}\right)^{p} .
$$

Other corollaries of the results we prove in Section 2 are given in Section 3, including applications to new congruences for the sums of squares functions, as well as a new approach to generating the odd-indexed sum of divisors function, $r_{4}(2 k+1) \equiv \sigma_{1}(2 k+1)$, through our new modified $q$-series generating function constructions.

We will also show in Section 3 that we can expand the generating function for the complete sequence of $\sigma_{1}(n)$, which is well-known to satisfy $\sigma_{1}(n)=-\left[q^{n-1}\right] d / d q\left[\log (q ; q)_{\infty}\right]$, using our new results applied to the series for the infinite $q$-Pochhammer symbol given by

$$
\begin{aligned}
&(q ; q)_{\infty}=1-q \times \sum_{i=1}^{\infty} \frac{(-1)^{i-1} q^{(9 i-2)(i-1) / 2}\left(q^{3} ; q^{3}\right)_{i-1}}{\left.\sum_{0 \leq j \leq n<2 i}\left[\begin{array}{l}
i \\
j
\end{array}\right]_{q^{3}}{ }^{i-1}{ }_{n-j}\right]_{q^{3}} q^{3 j} \cdot q^{(3 i-2) n}} \\
&-q^{2} \times \sum_{i=1}^{\infty} \frac{(-1)^{i-1} q^{(9 i+2)(i-1) / 2}\left(q^{3} ; q^{3}\right)_{i-1}}{\sum_{0 \leq j \leq n<2 i}\left[\begin{array}{l}
i \\
j
\end{array}\right]_{q^{3}}\left[\begin{array}{l}
i-1 \\
n-j
\end{array}\right]_{q^{3}} q^{3 j} \cdot q^{(3 i-1) n}}
\end{aligned}
$$

Another $q$-series expansion generating the partition function, $p(n)$, is similarly expanded through our new results as the reciprocal of the series in the previous equation. 
1.4. Other Applications of the New Results Proved in the Article. The results of the applications stated in Section 3 provide new series representations for generating functions of special sequences, including the sums of squares functions, $r_{p}(n)$, and the sum of divisors (divisor sigma) function, $\sigma_{1}(n)$, as well as new infinite series for the (higher-order) Jacobi theta functions, and other special case series results such as for the unilateral series expansion of the generalized two-variable Ramanujan theta function, $f(a, b)$, expanded by both its integral representation proved in [7] and our new series result in (6) as

$$
\begin{aligned}
f(a, b)=1 & +\int_{0}^{\infty} \frac{2 a e^{-t^{2} / 2}}{\sqrt{2 \pi}}\left[\frac{1-a \sqrt{a b} \cosh (\sqrt{\log (a b)} t)}{a^{3} b-2 a \sqrt{a b} \cosh (\sqrt{\log (a b)} t)+1}\right] d t \\
& +\int_{0}^{\infty} \frac{2 b e^{-t^{2} / 2}}{\sqrt{2 \pi}}\left[\frac{1-b \sqrt{a b} \cosh (\sqrt{\log (a b)} t)}{a b^{3}-2 b \sqrt{a b} \cosh (\sqrt{\log (a b)} t)+1}\right] d t \\
=1 & +\sum_{c \in\{a, b\}} \sum_{i=1}^{\infty} \frac{c \cdot(-1)^{i-1}(a b)^{(3 i-2)(i-1)}(a b ; a b)_{i-1} c^{2 i-2}}{\sum_{0 \leq j \leq n<2 i}\left[\begin{array}{l}
i \\
j
\end{array}\right]_{a b}\left[\begin{array}{l}
i-1 \\
n-j
\end{array}\right](a b)^{j} \cdot\left(-(a b)^{i-1} \cdot c\right)^{n}} .
\end{aligned}
$$

We also prove new convergent-function-based infinite $q$-series expansions of the Jacobi theta functions, $\vartheta_{i}(q, z)$, for $i:=1,2,3,4$ in Section 3.2. For example, the next special constant values of Ramanujan's $\varphi$-function and $\psi$-function cited as applications in [7], are expanded by the following new series and corresponding known integral representations from the reference:

$$
\begin{aligned}
\vartheta_{3}\left(0, e^{-5 \pi}\right) & \equiv \frac{\pi^{1 / 4}}{\Gamma\left(\frac{3}{4}\right)} \cdot \frac{\sqrt{5+2 \sqrt{5}}}{5^{3 / 4}} \\
& =1+\int_{0}^{\infty} \frac{e^{-t^{2} / 2}}{\sqrt{2 \pi}}\left[\frac{4 e^{5 \pi}\left(e^{10 \pi}-\cos (\sqrt{10 \pi} t)\right)}{e^{20 \pi}-2 e^{10 \pi} \cos (\sqrt{10 \pi} t)+1}\right] d t \\
& =1+2 e^{-5 \pi} \times \sum_{i=1}^{\infty} \frac{(-1)^{i-1} e^{-15 \pi i(i-1)}\left(e^{-10 \pi} ; e^{-10 \pi}\right)_{i-1}}{\sum_{0 \leq j \leq n<2 i}\left[\begin{array}{l}
i \\
j
\end{array}\right]_{e^{-10 \pi}}\left[\begin{array}{l}
i-1 \\
n-j
\end{array}\right] e^{-10 \pi} \times e^{-10 \pi \cdot j} \cdot\left(-e^{-5 \pi(2 i-1)}\right)^{n}} \\
\frac{e^{\pi / 8}}{2} \cdot \vartheta_{2}\left(0, e^{-\pi / 2}\right) & \equiv \frac{\pi^{1 / 4}}{\Gamma\left(\frac{3}{4}\right)} \cdot \frac{(\sqrt{2}+1)^{1 / 4} e^{\pi / 16}}{2^{7 / 16}} \\
& =\int_{0}^{\infty} \frac{e^{-t^{2} / 2}}{\sqrt{2 \pi}}\left[\frac{\cos \left(\sqrt{\frac{\pi}{2}} t\right)-e^{\pi / 4}}{\cos \left(\sqrt{\frac{\pi}{2}} t\right)-\cosh \left(\frac{\pi}{4}\right)}\right] d t \\
& =\sum_{i=1}^{\infty} \frac{(-1)^{i-1} e^{-\pi(3 i-2)(i-1) / 2}\left(e^{-\pi} ; e^{-\pi}\right)_{i-1}}{\sum_{0 \leq j \leq n<2 i}\left[\begin{array}{l}
i \\
j
\end{array}\right] e^{-\pi}\left[\begin{array}{l}
i-1 \\
n-j
\end{array}\right] e^{-\pi} \times e^{-\pi \cdot j} \cdot\left(-e^{-\pi(i-1)}\right)^{n}} .
\end{aligned}
$$

We cite other special case square series variants treated as notable examples in [7] as applications of Proposition 2.6 and Corollary 2.7 in Section 3 of the article below.

With the exception of the first examples of the new forms of the $q$-series generating functions enumerating the sequences, $r_{p}(n)$ and $\sigma_{1}(n)$, established in Section 3.1, we mostly follow the examples cited as noteworthy applications of the new results for square series integral representations proved in [7] in Section 3. Further special cases of a broader set of results generalizing (7) in terms of the Jacobi theta functions lead to other corollaries providing new series expansions of special products, new series representations of explicit constants involving real-valued multiples of reciprocals of the rational gamma function cases, $\Gamma\left(\frac{1}{4}\right), \Gamma\left(\frac{1}{3}\right)$, and $\Gamma\left(\frac{3}{4}\right)$, and integral-based representations of Mellin transforms defining known multiples of the Riemann zeta function, $\zeta(s)$, when $\Re(s)>2$.

1.5. Significance of the New Results in the Article. The new $q$-series expansions of special sequence generating functions and series for theta functions given by (6) are decidedly new in form and unlike other 
known series expansions for the special case examples we cite within the article. What is interesting to note about the proofs we obtain for these new series is that we apply the seemingly unrelated theory of J-fractions, and more general results on continued fractions, to a computationally-discovered sequence implicit to the typically combinatorially motivated definition of (1) to reach our new results for many special functions. As such, the rational $h^{\text {th }}$ partial sums with respect to $q$ and $z$ of the infinite series on the right-hand-side of (6) generate the expected series coefficients over $z$ (or $q$ if $z \equiv z(q)$ depends only on $q$ ) up to order $h+1$. The existence of the infinite series expansion follows by applying Pringsheim's theorem to our computationally-guided "guesses" at the correctness of the sequences we initially defined in (3) (see Remark 2.5 on page 11$)$.

Extensions of Our Computational Method. We note that our method of discovering the form of the infinite square series J-fraction expansion, $J_{\infty}^{[\mathrm{sq}]}(q, z)$, is easily replicated to find further analogous, if not sometimes related, new expansions for other special $q$-series generating functions. In the concluding remarks given in Section 4.2, we point out several additional computationally discovered sequence variants implicit to the J-fractions defined in (1) and provide a table of these identified component sequences generating a few notable $q$-series variants, including $q$-exponential functions, special cases of $q$-hypergeometric functions, and alternate known series related to the generating function for the partition function $p(n)$ (see Table 4 on page 18). We also provide as an example, but do not rigorously prove, another infinite $q$-series-like infinite sum analog to (6) which provides a generating function for the $q$-Pochhammer symbol, $(a ; q)_{n}=$ $(1-a)(1-a q) \cdots\left(1-a q^{n-1}\right)$. Another example which motivates further study of our methods described within this article, but that we do not explicitly cite closed-form sums for, provides generating functions for the divisor function, $d(n)=\sum_{d \mid n} 1$, in the form of ${ }^{2}$

$$
\sum_{n=1}^{\infty} d(n) q^{n}=(q ; q)_{\infty} \times \sum_{n=1}^{\infty} \frac{n q^{n}}{(q ; q)_{n}}
$$

where we can generate the terms of $n /(q ; q)_{n}$ through first-order derivatives of the newly identified series for $1 /(z ; q)_{\infty}$ with respect to $z$ defined in Section 4.2 .

\section{Proofs of the J-Fraction Representation}

\subsection{Exact Formulas for the Convergent Functions.}

Definition 2.1 ( $q$-Pochhammer Symbols and the $q$-Binomial Coefficients). For fixed non-zero $z, q \in \mathbb{C}$ and integers $n \geq 0$, we define the $q$-Pochhammer symbol, $(z ; q)_{n}$, as the product $[5, \S 17.2]$

$$
(z ; q)_{n}=\left\{\begin{array}{ll}
\prod_{j=0}^{n-1}\left(1-z q^{j}\right) & \text { if } n \geq 1 ; \\
1 & \text { if } n=0 .
\end{array} \quad\right. \text { (q-Pochhammer Symbol) }
$$

For $0 \leq m \leq n$, we define the Gaussian polynomial, or $q$-binomial coefficient, $\left[\begin{array}{c}n \\ m\end{array}\right]_{q}$, exactly as follows $[5$, $\S 17.2 ; \S 26.9]$ :

$$
\begin{aligned}
{\left[\begin{array}{c}
n \\
m
\end{array}\right]_{q}: } & \frac{(q ; q)_{n}}{(q ; q)_{m}(q ; q)_{n-m}} \\
& = \begin{cases}\frac{\left(1-q^{n}\right)\left(1-q^{n-1}\right) \cdots\left(1-q^{n-m+1}\right)}{(1-q)\left(1-q^{2}\right) \cdots\left(1-q^{m}\right)} & \text { if } 2 \leq m \leq n \\
1+q+q^{2}+\cdots+q^{n-1} & \text { if } m=1 ; \\
1 & \text { if } m=0 \\
0 & \text { otherwise }\end{cases}
\end{aligned}
$$

( $q$-Binomial Coefficients)

\footnotetext{
2 Additional identities of K. Dilcher from his article titled "Some q-series identities related to divisor functions" provide expansions of the series coefficients of $(q ; q)_{\infty} \times \sum_{n} n^{\alpha+1} q^{n} /(q ; q)_{n}$ when $\alpha \in \mathbb{Z}^{+}$. We can then generate these series by considering higher-order derivatives of our new J-fraction series for $1 /(z ; q)_{\infty}$ with respect to $z$ in the known Stirling number transformations proved in $[7, \S 2]$.
} 
The $q$-Pochhammer symbol is implicitly expanded in powers of $z$ by the $q$-binomial coefficients, $\left[\begin{array}{l}n \\ m\end{array}\right]_{q}$, as

$$
\begin{aligned}
(z ; q)_{n} & =(1-z)(1-z q) \cdots\left(1-z q^{n-1}\right) \\
& =\sum_{0 \leq i \leq n}\left[\begin{array}{c}
n \\
i
\end{array}\right]_{q} q^{\left(\begin{array}{l}
i \\
2
\end{array}\right)(-z)^{i} .}
\end{aligned}
$$

(q-Binomial Theorem)

The product-based definition of the $q$-Pochhammer symbol then implies that we have the following pair of triangular recurrences defining the $q$-binomial coefficients where $\left[\begin{array}{c}n \\ m\end{array}\right]_{q}=\left[\begin{array}{c}n \\ n-m\end{array}\right]_{q}$ for all $0 \leq m \leq n[5$, $\S 17.2(\mathrm{ii})]$ :

$$
\begin{aligned}
& {\left[\begin{array}{l}
n \\
m
\end{array}\right]_{q}=\left[\begin{array}{c}
n-1 \\
m-1
\end{array}\right]_{q}+q^{m}\left[\begin{array}{c}
n-1 \\
m
\end{array}\right]_{q}} \\
& {\left[\begin{array}{c}
n \\
m
\end{array}\right]_{q}=\left[\begin{array}{c}
n-1 \\
m
\end{array}\right]_{q}+q^{n-m}\left[\begin{array}{c}
n-1 \\
m-1
\end{array}\right]_{q} .}
\end{aligned}
$$

Proposition 2.2 (Denominator Convergent Function Formula). For fixed non-zero $z, q \in \mathbb{C}$ and integers $h \geq 0$, we have an exact representation of the denominator convergent functions, $\mathrm{Q}_{h}(q, z)$, expanded by the q-binomial coefficients, $\left[\begin{array}{c}n \\ m\end{array}\right]_{q^{2}}$, from Definition 2.1 in the following form:

$$
Q_{h}(q, z)=\sum_{0 \leq i \leq h}\left[\begin{array}{c}
h \\
i
\end{array}\right]_{q^{2}} q^{(2 h-1) i}(-z)^{i} .
$$

Proof. The proof follows from (5) and repeated applications of (8) by induction on $h$. In particular, we first notice that Table 2 implies that the right-hand-side formula stated by the proposition holds when $h:=0,1$. For some $h \geq 2$, we then assume that the right-hand-side formula for $\mathrm{Q}_{m}(q, z)$ in the proposition statement is correct for all $0 \leq m<h$, which, in particular, implies that the stated formula holds for $\mathrm{Q}_{h-1}(q, z)$ and $\mathrm{Q}_{h-2}(q, z)$ in the recurrence relation given in (5). If we shift the indices of summation, this implies that

$$
\begin{aligned}
& \mathrm{Q}_{h}(q, z)=\underbrace{\left[\begin{array}{c}
h-1 \\
0
\end{array}\right]_{q^{2}}+\left[\begin{array}{c}
h-1 \\
1
\end{array}\right]_{q^{2}} q^{2 h-3}(-z)-\left[z^{1}\right]\left(\sum_{1 \leq i \leq h}\left[\begin{array}{c}
h-1 \\
i-1
\end{array}\right]_{q^{2}} q^{(2 h-3)(i-1)}(-1)^{i-1} z^{i}\right) \cdot c_{h}^{[\mathrm{sq}]}(q) z}_{:=\mathrm{IC}_{h, 01}(q, z)} \\
& +\sum_{2 \leq i \leq h}\left[\left[\begin{array}{c}
h-1 \\
i
\end{array}\right]_{q^{2}} q^{-2 i}+\left(q^{4 h-3}+q^{4 h-5}-q^{2 h-3}\right) q^{-2 i}\left[\begin{array}{c}
h-1 \\
i-1
\end{array}\right]_{q^{2}}\right. \\
& \left.+\left(1-q^{2 h-2}\right) q^{2 h-4 i}\left[\begin{array}{c}
h-2 \\
i-2
\end{array}\right]_{q^{2}}\right]^{(2 h-1) i}(-z)^{i}
\end{aligned}
$$

We can show that $\operatorname{IC}_{h, 01}(q, z)=1+\left[\begin{array}{l}h \\ 1\end{array}\right]_{q^{2}} q^{2 h-1}(-z)$ by expanding the first three terms in the last equation as

$$
\begin{aligned}
\mathrm{IC}_{h, 01}(q, z) & =\left[\begin{array}{c}
h-1 \\
0
\end{array}\right]_{q^{2}}+\left[\begin{array}{c}
h-1 \\
1
\end{array}\right]_{q^{2}} q^{2 h-3}(-z)-\left[\begin{array}{c}
h-1 \\
0
\end{array}\right]_{q^{2}} q^{2 h-3} z\left(q^{2 h}+q^{2 h-2}-1\right) \\
& =1-q^{2 h-1} z \cdot\left[\left[\begin{array}{c}
h-1 \\
1
\end{array}\right]_{q^{2}}+q^{2 h}+q^{2 h-2}-1\right] \cdot q^{-2} \\
& =1-q^{2 h-1} z \times q^{-2} \cdot\left(1+q^{2}+q^{4}+\cdots+q^{2(h-2)}+q^{2 h-2}+q^{2 h}-1\right) \\
& =1+\left[\begin{array}{c}
h-1 \\
1
\end{array}\right]_{q^{2}} q^{2 h-1}(-z) .
\end{aligned}
$$

We complete the proof by simplifying the coefficients of the powers of $(-z)^{i}$ for $2 \leq i \leq h$ on the righthand-side of (i). More precisely, we show that for $i \geq 2$, we have the next equation in (ii), which we see 
completes the proof of our stated result.

$$
\left[\begin{array}{c}
h \\
i
\end{array}\right]_{q^{2}}=\left[\begin{array}{c}
h-1 \\
i
\end{array}\right]_{q^{2}} q^{-2 i}+\left(q^{2 h}+q^{2 h-2}-1\right) q^{-2 i}\left[\begin{array}{c}
h-1 \\
i-1
\end{array}\right]_{q^{2}}+\left(1-q^{2 h-2}\right) q^{2 h-4 i}\left[\begin{array}{c}
h-2 \\
i-2
\end{array}\right]_{q^{2}}
$$

We will require the next few lemmas following immediately as one and two-line consequences of the two recurrence relations for the $q$-binomial coefficients given in (8).

$$
\begin{aligned}
& q^{-2 i}\left(q^{2 h-2}-1\right)\left[\begin{array}{c}
h-2 \\
i-1
\end{array}\right]_{q^{2}}=q^{-2 i}\left(q^{2 h-2}-1\right)\left[\left[\begin{array}{c}
h-1 \\
i-1
\end{array}\right]_{q^{2}}-q^{2(h-i)}\left[\begin{array}{c}
h-2 \\
i-2
\end{array}\right]_{q^{2}}\right] \\
& q^{2(h-i)}\left[\begin{array}{c}
h-1 \\
i-1
\end{array}\right]_{q^{2}}=\left[\begin{array}{l}
h \\
i
\end{array}\right]_{q^{2}}-\left[\begin{array}{c}
h-1 \\
i
\end{array}\right]_{q^{2}} \\
& q^{2(h-1-i)}\left[\begin{array}{c}
h-2 \\
i-1
\end{array}\right]_{q^{2}}=\left[\begin{array}{c}
h-1 \\
i
\end{array}\right]_{q^{2}}-\left[\begin{array}{c}
h-2 \\
i
\end{array}\right]_{q^{2}} \\
& q^{-2 i}\left[\begin{array}{c}
h-1 \\
i
\end{array}\right]_{q^{2}}=q^{-2 i}\left[\begin{array}{c}
h-2 \\
i-1
\end{array}\right]_{q^{2}}+\left[\begin{array}{c}
h-2 \\
i
\end{array}\right]_{q^{2}} \\
& q^{2(h-i)}\left[\begin{array}{c}
h-1 \\
i-1
\end{array}\right]_{q^{2}}=\left[\begin{array}{c}
h \\
i
\end{array}\right]_{q^{2}}-\left[\begin{array}{c}
h-1 \\
i
\end{array}\right]_{q^{2}}
\end{aligned}
$$

We then proceed by simplifying the right-hand-side of (ii), denoted in shorthand notation by RHS[(ii)], as follows:

$$
\begin{aligned}
\operatorname{RHS}[(i i)] & =q^{-2 i}\left[\begin{array}{c}
h-1 \\
i
\end{array}\right]_{q^{2}}+q^{2(h-i)}\left[\begin{array}{c}
h-1 \\
i-1
\end{array}\right]_{q^{2}}+q^{-2 i}\left(q^{2 h-2}-1\right)\left[\begin{array}{c}
h-2 \\
i-1
\end{array}\right]_{q^{2}} & & \text { by (iii) } \\
& =\left[\begin{array}{c}
h-2 \\
i
\end{array}\right]_{q^{2}}+q^{2(h-i)}\left[\begin{array}{c}
h-1 \\
i-1
\end{array}\right]_{q^{2}}+q^{2(h-1-i)}\left[\begin{array}{c}
h-2 \\
i-1
\end{array}\right]_{q^{2}} & & \text { by (vi) } \\
& =\left[\begin{array}{c}
h \\
i
\end{array}\right]_{q^{2}} & & \text { by (iv), (v), and (vii). }
\end{aligned}
$$

Remark 2.3 (A Few Immediate Consequences). The formula for the exact expansion of the convergent functions, $\mathrm{Q}_{h}(q, z)$, for each $h \geq 1$ proved in Proposition 2.2 provides several immediate identities for the $h^{\text {th }}$ convergent functions, $\operatorname{Conv}_{h}(q, z)$, expressed through the denominator convergent functions $[5, c f$. $\S 1.12(\mathrm{ii})]$. In particular, for the $h^{\text {th }}$ modulus sequence, $\lambda_{h}(q):=a_{2} a_{3} \cdots a_{h+1}$ with $a_{h}:=\mathrm{ab}_{h}^{[\mathrm{sq}]}(q) \cdot z^{2}$, in $[1, \S 3]$ we have the following recurrence relation and exact expansion of the $h^{\text {th }}$ convergent function, $\operatorname{Conv}_{h}(q, z)$ :

$$
\begin{aligned}
\operatorname{Conv}_{h}(q, z) & =\operatorname{Conv}_{h-1}(q, z)+\frac{(-1)^{h-1} q^{(3 h-4)(h-1)}\left(q^{2} ; q^{2}\right)_{h-1} z^{2 h-2}}{\mathrm{Q}_{h-1}(q, z) \mathrm{Q}_{h}(q, z)} \\
& =\sum_{1 \leq i \leq h} \frac{(-1)^{i-1} q^{(3 i-4)(i-1)}\left(q^{2} ; q^{2}\right)_{i-1} z^{2 i-2}}{\mathrm{Q}_{i-1}(q, z) \mathrm{Q}_{i}(q, z)} .
\end{aligned}
$$

We are interested in the forms of the $q$-series expansions of the convergent functions, $\operatorname{Conv}_{h}\left(q, q^{2}\right)$, in the applications discussed in Section 3 of the article below. Notice that Proposition 2.2 applied to the forms of the previous equation implies that we have the exact formulas given by

$$
\begin{aligned}
\operatorname{Conv}_{h}\left(q, q^{2}\right) & =\sum_{1 \leq i \leq h} \frac{(-1)^{i-1} q^{3 i(i-1)}\left(q^{2} ; q^{2}\right)_{i-1}}{\mathrm{Q}_{i-1}\left(q, q^{2}\right) \mathrm{Q}_{i}\left(q, q^{2}\right)} \\
& =\sum_{1 \leq i \leq h} \frac{(-1)^{i-1} q^{3 i(i-1)}\left(q^{2} ; q^{2}\right)_{i-1}}{\sum_{0 \leq n<2 i}\left(\sum_{0 \leq j \leq n}\left[\begin{array}{l}
i \\
j
\end{array}\right]_{q^{2}}\left[\begin{array}{l}
i-1 \\
n-j
\end{array}\right]_{q^{2}} q^{2 j}\right)\left(-q^{2 i-1}\right)^{n}} .
\end{aligned}
$$


Proposition 2.4 (Numerator Convergent Function Formula). For fixed non-zero $z, q \in \mathbb{C}$ and integers $h \geq 0$, we have an exact representation of the numerator convergent functions, $\mathrm{P}_{h}(q, z)$, expanded in the following form:

$$
P_{h}(q, z)=\sum_{0 \leq n<h}\left(\sum_{0 \leq i \leq n}\left[\begin{array}{c}
h \\
i
\end{array}\right]_{q^{2}} q^{(2 h-1) i}(-1)^{i} q^{(n-i)^{2}}\right) z^{n} .
$$

Proof. If we let $C_{h, h}(q):=\left[z^{n}\right] \mathrm{P}_{h}(q, z)$, we see immediately from (5) that we have a recurrence relation for these coefficients given by

$$
C_{h, n}(q)=C_{h-1, n}(q)-q^{2 h-3}\left(q^{2 h}+q^{2 h-2}-1\right) C_{h-1, n-1}(q)-q^{6 h-10}\left(q^{2 h-2}-1\right) C_{h-2, n-2}(q) .
$$

We must prove that for all $h \geq 2$ and $0 \leq k<h$, we have the following result:

$$
C_{h, k}(q)=\sum_{0 \leq i \leq k}\left[\begin{array}{l}
h \\
i
\end{array}\right]_{q^{2}} q^{(2 h-1) i}(-1)^{i} q^{(k-i)^{2}} .
$$

We proceed by induction on $n$. Since $C_{n, k}(q) \equiv 0$ whenever $k<0$, we see easily from (10) that we have formulas for all $h \geq 2$ in the first two cases of $C_{h, n}$ when $n=0,1$ given by

$$
\begin{aligned}
C_{h, 0}(q) & =1 \\
C_{h, 1}(q) & =C_{h-1,1}(q)-q^{2 h-3}\left(q^{2 h}+q^{2 h-2}-1\right) \cdot 1 \\
& =-\sum_{i=2}^{h} q^{2 i-3}\left(q^{2 i}+q^{2 i-2}-1\right) \\
& =q-q^{2 h-1}\left(1+q^{2}+q^{4}+\cdots+q^{2 h-2}\right),
\end{aligned}
$$

each of which coincide with the formula in (11) whenever $h \geq 2$. Let $n \geq 2$ and suppose that (11) is true for all $0 \leq k \leq n-1$ when $h \geq 2$. We first show that

$$
C_{h, n}(q)-C_{h-1, n}(q)=\sum_{i=1}^{n}\left[\begin{array}{c}
h-1 \\
i-1
\end{array}\right]_{q^{2}}\left(q^{2 h}+q^{2 h-2 i}-1\right) q^{(2 h-3) i}(-1)^{i} q^{(n-i)^{2}} .
$$

To prove the formula given by the last result, we expand the left-hand-side of the previous equation according to (10) as follows:

$$
\begin{aligned}
C_{h, n}(q)-C_{h-1, n}(q)= & -q^{2 h-3}\left(q^{2 h}+q^{2 h-2}-1\right) C_{h-1, n-1}(q)-q^{6 h-10}\left(q^{2 h-2}-1\right) C_{h-2, n-2}(q) \\
= & -q^{2 h-3}\left(q^{2 h}+q^{2 h-2}-1\right) \sum_{i=0}^{n-1}\left[\begin{array}{c}
h-1 \\
i
\end{array}\right]_{q^{2}} q^{(2 h-3) i}(-1)^{i} q^{(n-1-i)^{2}} \\
& -q^{6 h-10}\left(q^{2 h-2}-1\right) \sum_{i=0}^{n-1}\left[\begin{array}{c}
h-2 \\
i
\end{array}\right]_{q^{2}} q^{(2 h-5) i}(-1)^{i} q^{(n-2-i)^{2}} \\
= & -\left(q^{2 h}+q^{2(h-1)}-1\right)\left[\begin{array}{c}
h-1 \\
0
\end{array}\right]_{q^{2}} q^{2 h-3} q^{(n-1)^{2}} \\
& +\sum_{i=2}^{n}\left(\begin{array}{l}
\left(q^{2 h}+q^{2 h-2}-1\right)\left[\begin{array}{c}
h-1 \\
i-1
\end{array}\right]_{q^{2}}-q^{2 h-2 i}\left(q^{2 h-2}-1\right)\left[\begin{array}{c}
h-2 \\
i-2
\end{array}\right]_{q^{2}}
\end{array}\right) \times \\
& \times q^{(2 h-3) i}(-1)^{i} q^{(n-i)^{2}} .
\end{aligned}
$$

It suffices to show that for any $h \geq 2$ and all $2 \leq i \leq n<h$, we have that

$$
\left[\begin{array}{c}
h-1 \\
i-1
\end{array}\right]_{q^{2}}\left(q^{2 h}+q^{2 h-2 i}-1\right)=\left(q^{2 h}+q^{2 h-2}-1\right)\left[\begin{array}{c}
h-1 \\
i-1
\end{array}\right]_{q^{2}}-q^{2 h-2 i}\left(q^{2 h-1}-1\right)\left[\begin{array}{c}
h-2 \\
i-2
\end{array}\right]_{q^{2}} .
$$


We complete the proof by proving that (12) is correct whenever $h \geq 2$ and $2 \leq i \leq n<h$ using the formulas for the $q$-binomial coefficients given in terms of the $q$-Pochhammer symbol in Definition 2.1 as follows:

$$
\begin{aligned}
\operatorname{RHS}[(12)] & =\left(q^{2 h}+q^{2 h-2}-1\right)\left[\begin{array}{c}
h-1 \\
i-1
\end{array}\right]_{q^{2}}-q^{2 h-2 i}\left(q^{2 h-1}-1\right)\left[\begin{array}{c}
h-2 \\
i-2
\end{array}\right]_{q^{2}} \\
& =\left(q^{2 h}+q^{2 h-2}-1\right) \frac{\left(q^{2} ; q^{2}\right)_{h-1}}{\left(q^{2} ; q^{2}\right)_{i-1}\left(q^{2} ; q^{2}\right)_{h-i}}-\frac{q^{2 h-2 i}\left(q^{2(h-1)}-1\right)\left(q^{2} ; q^{2}\right)_{h-2}}{\left(q^{2} ; q^{2}\right)_{i-2}\left(q^{2} ; q^{2}\right)_{h-i}} \\
& =\left[\begin{array}{c}
h-1 \\
i-1
\end{array}\right]_{q^{2}}\left(\left(q^{2 h}+q^{2 h-2}-1\right)+q^{2 h-2 i}\left(1-q^{2 i-2}\right)\right) \\
& =\left[\begin{array}{c}
h-1 \\
i-1
\end{array}\right]_{q^{2}}\left(q^{2 h}+q^{2 h-2 i}-1\right)
\end{aligned}
$$

Thus we have that

$$
\begin{aligned}
C_{h, n}(q) & =C_{h-1, n}(q)+\sum_{i=1}^{n}\left[\begin{array}{c}
h-1 \\
i-1
\end{array}\right]_{q^{2}}\left(q^{2 h}+q^{2 h-2 i}-1\right) q^{(2 h-3) i}(-1)^{i} q^{(n-i)^{2}} \\
& =\sum_{j=1}^{h} \sum_{i=1}^{n}\left[\begin{array}{l}
j-1 \\
i-1
\end{array}\right]_{q^{2}}\left(q^{2 j}+q^{2 j-2 i}-1\right) q^{(2 j-3) i}(-1)^{i} q^{(n-i)^{2}}
\end{aligned}
$$

and so to complete the proof, it suffices to show that for all integers $k \geq 1$ and all $1 \leq i \leq k$, we have the next result which we will prove by induction on $k(h)$ below.

$$
\sum_{j=1}^{k}\left[\begin{array}{l}
j-1 \\
i-1
\end{array}\right]_{q^{2}}\left(q^{2 j}+q^{2 j-2 i}-1\right) q^{(2 j-3) i}=\left[\begin{array}{c}
k \\
i
\end{array}\right]_{q^{2}} q^{(2 k-1) i}
$$

When $k=i=1$, we see that

$$
\left[\begin{array}{l}
0 \\
0
\end{array}\right]_{q^{2}}\left(q^{2}+1-1\right) \cdot q^{-1}=\left[\begin{array}{l}
1 \\
1
\end{array}\right]_{q^{2}} \cdot q=q .
$$

Next, we suppose that (13) is true for all $k<h$ when $h \geq 2$ and rewrite (13) according to our hypothesis when $k=h$ as follows:

$$
\begin{aligned}
& \operatorname{LHS}[(13)]=\sum_{j=1}^{h}\left[\begin{array}{l}
j-1 \\
i-1
\end{array}\right]_{q^{2}}\left(q^{2 j}+q^{2 j-2 i}-1\right) q^{(2 j-3) i} \\
& =q^{(2 h-1) i} \cdot q^{-2 i}\left(\left[\begin{array}{c}
h-1 \\
i
\end{array}\right]_{q^{2}}+\left[\begin{array}{c}
h-1 \\
i-1
\end{array}\right]_{q^{2}} q^{2(h-i)}-\left(1-q^{2 h}\right)\left[\begin{array}{c}
h-1 \\
i-1
\end{array}\right]_{q^{2}}\right) \\
& =q^{(2 h-1) i} \cdot q^{-2 i}\left(\left[\begin{array}{c}
h \\
i
\end{array}\right]_{q^{2}}-\left(1-q^{2 h}\right)\left[\begin{array}{c}
h-1 \\
i-1
\end{array}\right]_{q^{2}}\right) \\
& =q^{(2 h-1) i} \cdot q^{-2 i}\left[\begin{array}{c}
h \\
i
\end{array}\right]_{q^{2}}\left(1-\frac{\left(1-q^{2 h}\right)\left(1-q^{2 i}\right)}{\left(1-q^{2 h}\right)}\right) \\
& =\left[\begin{array}{c}
h \\
i
\end{array}\right]_{q^{2}} q^{(2 h-1) i} .
\end{aligned}
$$

\subsection{Proof of the Main Theorem.}

Proof of Theorem 1.2. Since both of $\mathrm{P}_{h}(q, z)$ and $\mathrm{Q}_{h}(q, z)$ finite-degree polynomials in $z$ (and in $q$ ), we see that for all $h \geq 1$, the $h^{\text {th }}$ convergent functions, $\operatorname{Conv}_{h}(q, z)$, are rational functions of $z$. This fact, together with the observation that $\operatorname{deg}_{z}\left\{\mathrm{Q}_{h}(q, z)\right\}=h$ for all $h \geq 0$, implies that we have $h$-order finite difference 
equations for the coefficients of $\operatorname{Conv}_{h}(q, z) \equiv \mathrm{P}_{h}(q, z) / \mathrm{Q}_{h}(q, z)$ of the following form [4, cf. §2]:

$$
\left[z^{n}\right] \operatorname{Conv}_{h}(q, z)=-\sum_{0<i \leq \min (n, h)}\left[z^{i}\right] \mathrm{Q}_{h}(q, z) \cdot\left[z^{h-i}\right] \operatorname{Conv}_{h}(q, z)+\left[z^{n}\right] \mathrm{P}_{h}(q, z) .
$$

We first notice that for any functions, $F(z)$ and $G(z)$, such that the ratio of these two functions has a power series expansion in $z$ about 0 , we have that $\left[z^{0}\right] F(z) / G(z)=F(0) / G(0)$, which by Proposition 2.2 and Proposition 2.4 then implies that $\left[z^{0}\right] \operatorname{Conv}_{h}(q, z)=1$ for all $h \geq 1$. Then if we assume by induction that for fixed $h \geq 1$ and all $0 \leq i<n$ we have that $\left[z^{i}\right] \operatorname{Conv}_{h}(q, z)=q^{i^{2}}$, we can use the two propositions to rewrite (i) of the previous equation in the following forms when $0 \leq n<h$ :

$$
\begin{aligned}
{\left[z^{n}\right] \operatorname{Conv}_{h}(q, z)=} & -\sum_{1 \leq i \leq n}\left[\begin{array}{l}
h \\
i
\end{array}\right]_{q^{2}} q^{(2 h-1) i}(-1)^{i} q^{(n-i)^{2}} \\
& +\left(\sum_{0 \leq i \leq n}\left[\begin{array}{c}
h \\
i
\end{array}\right]_{q^{2}} q^{(2 h-1) i}(-1)^{i} q^{(n-i)^{2}}\right) \cdot[n<h]_{\delta}, \text { where } 0 \leq n-i<h \forall n \leq h \\
= & \left.\left\{\begin{array}{c}
h \\
i
\end{array}\right]_{q^{2}} q^{(2 h-1) i}(-1)^{i} q^{(n-i)^{2}}\right\}\left.\right|_{i=0} \\
= & q^{n^{2}} .
\end{aligned}
$$

To prove that $\left[z^{h}\right] \operatorname{Conv}_{h}(q, z)=q^{n^{2}}$ when $n \equiv h$, we expand (i) using the known $q$-binomial theorem identity for the $q$-Pochhammer symbol, $(z ; q)_{h}$, stated in Definition 2.1 as follows [5, §17.2(iii)]:

$$
\begin{aligned}
{\left[z^{n}\right] \operatorname{Conv}_{h}(q, z) } & =-\sum_{1 \leq i \leq h}\left[\begin{array}{c}
h \\
i
\end{array}\right]_{q^{2}} q^{(2 h-1) i}(-1)^{i} q^{(n-i)^{2}} \\
& =-q^{n^{2}} \times \sum_{1 \leq i \leq h}\left[\begin{array}{c}
h \\
i
\end{array}\right]_{q^{2}} q^{(2 h-2 n-1) i}(-1)^{i} q^{i^{2}} \\
& =-q^{n^{2}} \times \sum_{1 \leq i \leq h}\left[\begin{array}{c}
h \\
i
\end{array}\right]_{q^{2}}(-1)^{i} q^{2\left(\begin{array}{c}
i \\
2
\end{array}\right)} \\
& =-q^{n^{2}}\left[\left(1 ; q^{2}\right)_{h}-\left[\begin{array}{c}
h \\
0
\end{array}\right]_{q^{2}}\right] \\
& =q^{n^{2}}
\end{aligned}
$$

Remark 2.5 (Convergence and Stronger Statements of the Theorem). We note that we actually have a stronger result, which is that $\left[z^{n}\right] \operatorname{Conv}_{h}(q, z)=q^{n^{2}}$ for all $0 \leq n<2 h$ when $h \geq 1$, though we only needed to prove the weaker statement given in Theorem 1.2 above to prove the correctness of our new square series J-fraction expansions defined in (2) of the introduction. We can strengthen the statement of the theorem by adding that when $0<|q|,|z|<1, J_{\infty}^{[\mathrm{sq}]}(q, z):=\lim _{h \rightarrow \infty} \operatorname{Conv}_{h}(q, z)<\infty$, exists, and moreover, the corresponding infinite sum in (9) converges uniformly to the ordinary form of the geometric square series we have already proved convergent integral representations for in [7]. The proof of this statement is given by applying Pringsheim's theorem on the convergence of more general continued fractions when $\left|a_{h}\right|:=\left|z^{2} \cdot q^{6 h-10}\left(q^{2 h-2}-1\right)\right|$ and $\left|b_{h}\right|:=\left|1-q^{2 h-3}\left(q^{2 h}+q^{2 h-2}-1\right) \cdot z\right|$ where $|q|,|z|<1$, so that $\left|b_{h}\right|-1 \geq\left|a_{h}\right|$ for all $h \geq 2$, which then implies that $\left|\operatorname{Conv}_{h}(q, z)\right|<1$ for all large enough $h[5, \S 1.12(\mathrm{v})][8, \S \mathrm{II}]$.

We can then use the Weierstrass $M$-test to prove that we can differentiate the limiting case of the infinite $q$-series in (9) termwise with respect to $z$. We use these two facts, namely 1 ) that the limit function $J_{\infty}^{[\mathrm{sq}]}(q, z)$ - and so the new infinite square series representation in $(9)$ - exists as $h \rightarrow \infty$; and 2$)$ that this series is uniformly convergent in $z$, to prove new forms of infinite $q$-series expansions of these ordinary square series when $|z|<1$, or when the parametric $z:= \pm c \cdot q^{p m}$ depends on powers of the auxiliary square series parameter $|q|<1$. 


\subsection{Consequences of the Main Theorem.}

Proposition 2.6 (New Infinite Series Representations of the Geometric Square Series). For fixed $q, z \in \mathbb{C}$ defined such that $0<|q|,|z|<1$, we have the following infinite series representation of the ordinary geometric square series, $J_{\infty}^{[\mathrm{sq}]}(q, z)$ :

$$
J_{\infty}^{[\mathrm{sq}]}(q, z)=\sum_{i=1}^{\infty} \frac{(-1)^{i-1} q^{(3 i-4)(i-1)}\left(q^{2} ; q^{2}\right)_{i-1} z^{2 i-2}}{\sum_{0 \leq j \leq n<2 i}\left[\begin{array}{l}
i \\
j
\end{array}\right]_{q^{2}}\left[\begin{array}{l}
i-1 \\
n-j
\end{array}\right]_{q^{2}} q^{2 j} \cdot\left(-q^{2 i-3} z\right)^{n}} .
$$

Proof. We employed known expansions of the convergents to arbitrary continued fractions cited for reference in $\left[5, \S 1.12(\right.$ ii) $]$ to obtain the finite sum representations of $\operatorname{Conv}_{h}(q, z)$ stated in (9), which it is easy to see hold for each finite $h \geq 1$. We know by the discussion above in Remark 2.5 that the infinite limit, $\lim _{h \rightarrow \infty} \operatorname{Conv}_{h}(q, z)$, exists, which implies that we may form a new infinite series expansion for $J_{\infty}^{[\mathrm{sq}]}(q, z)$ by letting $h \longrightarrow \infty$ in (9). We then complete the proof by forming a discrete convolution of the coefficients of the polynomial expansions for the convergent denominator functions, $\mathrm{Q}_{h}(q, z)$, in $z$ proved as in the formula from Proposition 2.2.

Another class of series representations for special functions involves taking the derivatives of $J_{\infty}^{[\mathrm{sq}]}(q, z)$ termwise with respect to $z$. The first-order case of such derivatives of our special square series expansions with a corresponding integral representation proved in [7] given by

$$
\begin{aligned}
\vartheta_{0,1}(a, b ; q, z): & =\sum_{n}(a n+b) q^{n^{2}} z^{n} \\
& =\int_{0}^{\infty} \frac{2 a c z e^{-t^{2} / 2}}{\sqrt{2 \pi}}\left[\frac{\left(c^{2} z^{2}+1\right) \cosh (t \sqrt{2 \log (q)})-2 c z}{\left(c^{2} z^{2}-2 c z \cosh (t \sqrt{2 \log (q)})+1\right)^{2}}\right] d t+b \cdot J_{\infty}^{[\mathrm{sq}]}(q, z),
\end{aligned}
$$

arises frequently in applications, and so is explicitly considered as the particular case in the next corollary. Other particular explicit higher-order derivative expansions analogous to the formula cited in the previous equation for $\vartheta_{0, m}(a, b ; q, z)$ when $m \geq 2$ is integer-valued are of course similarly easy to obtain directly by repeated differentiation of the standard quotient rule.

Corollary 2.7 (New Series Representations for Derivatives of the Geometric Square Series). Suppose that $q, z \in \mathbb{C}$ are defined such that $0<|q|,|z|<1$, and that the component series functions, $f_{q, i}(z)$ and $g_{q, i}(z)$, are defined for integers $i \geq 1$ by the next formulas.

$$
\begin{aligned}
f_{q, i}(z) & =(-1)^{i-1} q^{(3 i-4)(i-1)}\left(q^{2} ; q^{2}\right)_{i-1} z^{2 i-2} \\
f_{q, i}^{\prime}(z) & =(2 i-2) \cdot(-1)^{i-1} q^{(3 i-4)(i-1)}\left(q^{2} ; q^{2}\right)_{i-1} z^{2 i-3} \\
g_{q, i}(z) & =\sum_{0 \leq j \leq n<2 i}\left[\begin{array}{l}
i \\
j
\end{array}\right]_{q^{2}}\left[\begin{array}{l}
i-1 \\
n-j
\end{array}\right]_{q^{2}} q^{2 j} \cdot\left(-q^{2 i-3} z\right)^{n} \\
g_{q, i}^{\prime}(z) & =\sum_{0 \leq j \leq n<2 i}\left[\begin{array}{l}
i \\
j
\end{array}\right]_{q^{2}}\left[\begin{array}{l}
i-1 \\
n-j
\end{array}\right]_{q^{2}} n \cdot q^{2 j} \cdot\left(-q^{2 i-3}\right)^{n} z^{n-1}
\end{aligned}
$$

Then for fixed scalars $a, b \in \mathbb{C}$ (not both zero), we have a new infinite series for the ordinary square series generating functions, $\vartheta_{0,1}(a, b ; q, z)$, in the form of

$$
\vartheta_{0,1}(a, b ; q, z)=a z \times \sum_{i=1}^{\infty} \frac{f_{q, i}^{\prime}(z) g_{q, i}(z)-f_{q, i}(z) g_{q, i}^{\prime}(z)}{\left[g_{q, i}(z)\right]^{2}}+b \cdot J_{\infty}^{[\mathrm{sq}]}(q, z) .
$$

Proof. The result follows directly from the quotient rule applied to Proposition 2.6. We note that we may differentiate the functions of $z$ in the series from the previous proposition term by term over each $i$ since we have already shown in Remark 2.5 that this limiting case of the $h^{\text {th }}$ convergent function series in (9) converges uniformly as a function of $z$. 


\section{Applications}

\begin{tabular}{|l|l|}
\hline$h$ & $1+2 q \cdot \operatorname{Conv}_{h}\left(q, q^{2}\right)$ \\
\hline 1 & $\frac{(1+q)\left(-1-q+q^{2}\right)}{(-1+q)\left(1+q+q^{2}\right)}$ \\
2 & $\frac{(1+q)^{2}\left(-1-q-2 q^{3}-q^{4}-q^{6}+q^{7}\right)}{\left(1+q^{2}\right)\left(-1-q-q^{4}+q^{6}+q^{7}\right)}$ \\
3 & $\frac{(1+q)^{3}\left(1-q+q^{2}\right)\left(1+q+q^{3}+q^{5}+q^{6}-q^{7}+q^{8}-q^{9}-q^{11}-2 q^{12}-q^{14}+q^{15}\right)}{(-1+q)\left(1+q^{2}\right)\left(1+q+q^{2}\right)\left(1+q+q^{2}+q^{3}+q^{4}+q^{5}+q^{6}\right)\left(-1+q^{2}-q^{3}-q^{4}+q^{5}-q^{7}+q^{9}\right)}$ \\
4 & $\frac{(1+q)^{4}\left(1-q+q^{2}\right)\left(1-q^{2}+2 q^{3}+q^{6}+2 q^{8}-q^{9}+q^{10}+q^{12}-2 q^{14}+3 q^{15}-2 q^{16}-q^{18}-q^{21}+q^{22}-2 q^{23}+q^{24}\right)}{\left(1+q^{4}\right)\left(1+q+q^{3}+q^{4}+q^{6}+q^{7}+q^{8}+q^{9}-q^{13}-q^{14}-q^{15}-q^{16}-q^{17}-q^{18}-q^{19}-q^{20}-q^{21}+q^{25}+q^{26}\right)}$ \\
\hline
\end{tabular}

TABLE 3. Special Cases of the Convergent Functions, $\widetilde{\vartheta}_{3, h}(q):=1+2 q \cdot \operatorname{Conv}_{h}\left(q, q^{2}\right)$

3.1. New Generating Functions for the Sums of Squares Functions. The result proved in Proposition 2.6 implies that in limiting cases we also have new infinite series for the ordinary generating functions of $r_{p}(n)$ for $p \geq 2$ in the following forms:

$$
\vartheta_{3}(q)^{p}=\left(1+2 q \times \sum_{i=1}^{\infty} \frac{(-1)^{i-1} q^{3 i(i-1)}\left(q^{2} ; q^{2}\right)_{i-1}}{\sum_{0 \leq n<2 i}\left(\sum_{0 \leq j \leq n}\left[\begin{array}{l}
i \\
j
\end{array}\right]_{q^{2}}\left[\begin{array}{l}
i-1 \\
n-j
\end{array}\right]_{q^{2}} q^{2 j}\right)\left(-q^{2 i-1}\right)^{n}}\right)^{p} .
$$

For $0 \leq n \leq(2 h-1)^{2}$ (and occasionally for slightly larger $n$ ), we have that

$$
r_{p}(n)=\left[q^{n}\right]\left(1+2 q \cdot \operatorname{Conv}_{h}\left(q, q^{2}\right)\right)^{p} .
$$

Several special cases of the forms of the modified $h^{\text {th }}$ convergent functions, $\widetilde{\vartheta}_{3, h}(q):=1+2 q \cdot \operatorname{Conv}_{h}\left(q, q^{2}\right)$, are expanded in factored form in Table 3 . These special case convergent functions also satisfy expansions given by

$$
\begin{aligned}
& \widetilde{\vartheta}_{3,2}(q)=1+\frac{2(2+3 q)}{13\left(1+q^{2}\right)}-\frac{2\left(-2+8 q+12 q^{2}+5 q^{3}+12 q^{4}+18 q^{5}+3 q^{6}\right)}{13\left(-1-q-q^{4}+q^{6}+q^{7}\right)} \\
& \widetilde{\vartheta}_{3,3}(q)=1-\frac{4}{21(-1+q)}+\frac{2(3+4 q)}{25\left(1+q^{2}\right)}+\frac{2(-2+5 q)}{39\left(1+q+q^{2}\right)} \\
& +\frac{2\left(-31-41 q+82 q^{2}+44 q^{3}+90 q^{4}+66 q^{5}\right)}{301\left(1+q+q^{2}+q^{3}+q^{4}+q^{5}+q^{6}\right)} \\
& -\frac{2\left(-852+8364 q-2721 q^{2}-5830 q^{3}+17408 q^{4}+1596 q^{5}-10747 q^{6}+12927 q^{7}+5761 q^{8}\right)}{13975\left(-1+q^{2}-q^{3}-q^{4}+q^{5}-q^{7}+q^{9}\right)} \\
& \widetilde{\vartheta}_{3,4}(q)=1-\frac{2\left(16-64 q-q^{2}+4 q^{3}\right)}{257\left(1+q^{4}\right)} \\
& +\frac{\vartheta_{3,4}(q)}{\left(257\left(1+q+q^{3}+q^{4}+q^{6}+q^{7}+q^{8}+q^{9}-q^{13}-q^{14}-q^{15}-q^{16}-q^{17}-q^{18}-q^{19}-q^{20}-q^{21}+q^{25}+q^{26}\right)\right)},
\end{aligned}
$$

where the numerator polynomial, $\vartheta_{3,4}(q)$, is defined by

$$
\begin{aligned}
\vartheta_{3,4}(q):= & \left(2 \left(16+209 q+192 q^{2}+19 q^{3}+454 q^{4}+497 q^{5}+84 q^{6}+451 q^{7}+525 q^{8}+486 q^{9}+626 q^{10}\right.\right. \\
& +323 q^{11}+507 q^{12}+526 q^{13}-64 q^{14}-17 q^{15}+309 q^{16}+33 q^{17}-405 q^{18}-195 q^{19} \\
& \left.\left.-264 q^{20}-502 q^{21}-305 q^{22}-322 q^{23}-254 q^{24}+4 q^{25}\right)\right) .
\end{aligned}
$$

Remark 3.1 (Convergent Function Congruences Versus Generating Functions as Series Over q). Note that while the J-fraction properties listed in the references $[1,2,4,6]$ provide congruences for the coefficients of $\operatorname{Conv}_{h}(q, z)$ over $z$ modulo $h$ for integers $h \geq 2$, we are unable to find exact generating functions for the 
sums of squares functions, $r_{p}(n)(\bmod h)$, which are generated by corresponding powers series expansions over $q$ since $\left[z^{n}\right] \operatorname{Conv}_{h}(q, z) \neq q^{n^{2}}$ for $n \geq 2 h$. We instead adapt our expansions from the previous few equations to form partial generating functions in the next corollaries by reducing the polynomial numerator and denominator term coefficients modulo $h$. This procedure follows by the approach to generating the functions, $r_{p}(n)$, recursively for $0 \leq n<h$ by the finite difference equations implied by the rationality of $\widetilde{\vartheta}_{3, h}(q)$ in $q$ for all $h \geq 2$.

Corollary 3.2 (Generating Functions Enumerating $r_{p}(n)$ Modulo 3 ). For $0 \leq n \leq 36$ and integers $p \geq 2$, we have that

$$
r_{p}(n) \equiv\left[q^{n}\right]\left(1+\frac{1+2 q+2 q^{2}+q^{3}}{1+q+q^{2}+q^{3}+q^{4}+q^{5}+q^{6}}+\frac{2+q+q^{3}+2 q^{7}+2 q^{8}+2 q^{11}+q^{12}}{1+2 q^{9}+2 q^{11}+q^{14}}\right)^{p} \quad(\bmod 3) .
$$

Corollary 3.3 (Generating Functions Enumerating $r_{p}(n)$ Modulo 4 ). For $0 \leq n \leq 64$ and integers $p \geq 2$, we have that

$$
r_{p}(n) \equiv\left[q^{n}\right]\left(1+\frac{2 q^{2}}{1+q^{4}}+\frac{2 q+2 q^{3}+2 q^{5}+2 q^{7}+2 q^{8}+2 q^{11}+2 q^{12}+2 q^{15}+2 q^{16}+2 q^{17}+2 q^{18}+2 q^{19}+2 q^{22}}{1+q+q^{3}+q^{4}+q^{6}+q^{7}+q^{8}+q^{9}+3 q^{13}+3 q^{14}+3 q^{15}+3 q^{16}+3 q^{17}+3 q^{18}+3 q^{19}+3 q^{20}+3 q^{21}+q^{25}+q^{26}}\right)^{p} \quad(\bmod 4) .
$$

Remark 3.4 (Generating Functions Enumerating the Sum of Divsors Function). Since $r_{4}(2 k+1)=8$. $\sigma_{1}(2 k+1)$ for all integers $k \geq 0$, we may employ the first infinite $q$-series expansion given in this section to enumerate the sum of divisors function, $\sigma_{1}(n)=\sum_{d \mid n} d$, over odd positive integers $n$ as follows:

$$
\begin{aligned}
\sum_{k=0}^{\infty} \sigma_{1}(2 k+1) q^{2 k+1} & =\frac{1}{16} \cdot\left(\vartheta_{3}(q)^{4}-\vartheta_{3}(-q)^{4}\right) \\
& =\sum_{b= \pm 1} \frac{b}{16}\left(1+2 b q \times \sum_{i=1}^{\infty} \frac{(-1)^{i-1}(b q)^{3 i(i-1)}\left(q^{2} ; q^{2}\right)_{i-1}}{\sum_{0 \leq j \leq n<2 i}\left[\begin{array}{l}
i \\
j
\end{array}\right]_{q^{2}}\left[\begin{array}{l}
i-1 \\
n-j
\end{array}\right]_{q^{2}} q^{2 j}\left(-b q^{2 i-1}\right)^{n}}\right)^{4}
\end{aligned}
$$

We can also use the square series generating function results for $J_{\infty}^{[\mathrm{sq}]}(q, z) \equiv \operatorname{Conv}_{\infty}(q, z)$ from Remark 2.3 and Proposition 2.6 to prove new $q$-series expansions for the infinite $q$-Pochhammer product, $(q ; q)_{\infty}$, which generates the sum of divisors function as [5, §26.10(iii)]

$$
\sum_{n \geq 1} \sigma_{1}(n) q^{n}=-q \cdot d / d q\left[\log (q ; q)_{\infty}\right]
$$

Then we expand $(q ; q)_{\infty}$ in the generating function for $\sigma_{1}(n)$ in the previous equation by the unilateral series given by

$$
\begin{aligned}
(q ; q)_{\infty}= & 1-\sum_{n=0}^{\infty}(-1)^{n}\left(q^{(n+1)(3 n+2) / 2}+q^{(n+1)(3 n+4) / 2}\right) \\
= & 1-q \times \sum_{n=0}^{\infty}\left(-q^{5 / 2}\right)^{n} q^{3 n^{2} / 2}-q^{2} \times \sum_{n=0}^{\infty}\left(-q^{7 / 2}\right)^{n} q^{3 n^{2} / 2} \\
= & 1-q \times \sum_{i=1}^{\infty} \frac{(-1)^{i-1} q^{(9 i-2)(i-1) / 2}\left(q^{3} ; q^{3}\right)_{i-1}}{\sum_{0 \leq j \leq n<2 i}\left[\begin{array} { l } 
{ i } \\
{ j }
\end{array} q ^ { 3 } \left[\begin{array}{l}
i-1 \\
n-j
\end{array} q^{3} q^{3 j} \cdot q^{(3 i-2) n}\right.\right.} \\
& -q^{2} \times \sum_{i=1}^{\infty} \frac{(-1)^{i-1} q^{(9 i+2)(i-1) / 2}\left(q^{3} ; q^{3}\right)_{i-1}}{\sum_{0 \leq j \leq n<2 i}\left[\begin{array}{l}
i \\
j
\end{array}\right]_{q^{3}}\left[\begin{array}{l}
i-1 \\
n-j
\end{array}\right] q^{3} q^{3 j} \cdot q^{(3 i-1) n}}
\end{aligned}
$$

Similarly, the partition function, $p(n)$, is generated by the $q$-series expansion of the reciprocal of the previous series for $(q ; q)_{\infty}$. Other applications of the new infinite $q$-series provided by the limiting cases of these sums for the $h^{\text {th }}$ convergent functions are given in the next section. 
3.2. New Infinite Series for Special Theta Functions. The Jacobi theta functions, denoted by $\vartheta_{i}(u, q)$ for $i=1,2,3,4$, or by $\vartheta_{i}(u \mid \tau)$ when the nome $q \equiv \exp (\imath \pi \tau)$ satisfies $\Im(\tau)>0$, form another class of square series expansions of special interest in the applications considered in [7]. The classical forms of these theta functions satisfy the respective bilateral and corresponding asymmetric, unilateral Fourier-type square series expansions given by $[5, \S 20.2(\mathrm{i})]$

$$
\begin{aligned}
& \vartheta_{1}(u, q)=\sum_{n=-\infty}^{\infty} q^{\left(n+\frac{1}{2}\right)^{2}}(-1)^{n-1 / 2} e^{(2 n+1) \imath u} \quad=2 q^{1 / 4} \sum_{n=0}^{\infty} q^{n(n+1)}(-1)^{n} \sin ((2 n+1) u) \\
& \vartheta_{2}(u, q)=\sum_{n=-\infty}^{\infty} q^{\left(n+\frac{1}{2}\right)^{2}} e^{(2 n+1) \imath u} \\
& =2 q^{1 / 4} \sum_{n=0}^{\infty} q^{n(n+1)} \cos ((2 n+1) u) \\
& \vartheta_{3}(u, q)=\sum_{n=-\infty}^{\infty} q^{n^{2}} e^{2 n \imath u} \\
& =1+2 \sum_{n=1}^{\infty} q^{n^{2}} \cos (2 n u) \\
& \vartheta_{4}(u, q)=\sum_{n=-\infty}^{\infty} q^{n^{2}}(-1)^{n} e^{2 n \imath u} \\
& =1+2 \sum_{n=1}^{\infty} q^{n^{2}}(-1)^{n} \cos (2 n u) .
\end{aligned}
$$

Corollary 3.5 (New $q$-Series Expansions of the Jacobi Theta Functions). For $u \in \mathbb{R}$ and fixed $q \in \mathbb{C}$ such that $0<|q|<1$, we have new q-series representations of the Fourier series for the Jacobi theta functions expanded in the following forms:

$$
\begin{aligned}
& \vartheta_{1}(u, q)=\sum_{b= \pm 1} \frac{q^{1 / 4} e^{\imath b u}}{b \imath} \times\left[\sum_{i=1}^{\infty} \frac{(-1)^{i-1} q^{(3 i-4)(i-1)}\left(q^{2} ; q^{2}\right)_{i-1}\left(-e^{2 \imath b u} q\right)^{2 i-2}}{\sum_{0 \leq j \leq n<2 i}\left[\begin{array}{l}
i \\
j
\end{array}\right]_{q^{2}}\left[\begin{array}{l}
i-1 \\
n-j
\end{array}\right]_{q^{2}} q^{2 j} \cdot\left(q^{i-1} e^{\imath b u}\right)^{2 n}}\right] \\
& \vartheta_{2}(u, q)=\sum_{b= \pm 1} q^{1 / 4} e^{\imath b u} \times\left[\sum_{i=1}^{\infty} \frac{(-1)^{i-1} q^{(3 i-4)(i-1)}\left(q^{2} ; q^{2}\right)_{i-1}\left(q e^{2 \imath b u}\right)^{2 i-2}}{\sum_{b= \pm 1}\left[\begin{array}{l}
i \\
j
\end{array}\right]_{q^{2}}\left[\begin{array}{l}
i-1 \\
n-j
\end{array}\right]_{q^{2}} q^{2 j} \cdot\left(q^{i-1} e^{\imath b u}\right)^{2 n}}\right] \\
& \vartheta_{3}(u, q)=1+\sum_{b=2 i} q e^{2 \imath b u} \times\left[\sum_{i=1}^{\infty} \frac{(-1)^{i-1} q^{(3 i-4)(i-1)}\left(q^{2} ; q^{2}\right)_{i-1}\left(q e^{\imath b u}\right)^{4 i-4}}{\sum_{0 \leq j \leq n<2 i}\left[\begin{array}{l}
i \\
j
\end{array}\right]_{q^{2}}\left[\begin{array}{l}
i-1 \\
n-j
\end{array}\right]_{q^{2}} q^{2 j} \cdot\left(-q^{2 i-1} e^{2 \imath b u}\right)^{n}}\right] \\
& \vartheta_{4}(u, q)=1-\sum_{b= \pm 1} q e^{2 \imath b u} \times\left[\sum_{i=1}^{\infty} \frac{(-1)^{i-1} q^{(3 i-4)(i-1)}\left(q^{2} ; q^{2}\right)_{i-1}\left(-q e^{\imath b u}\right)^{4 i-4}}{\sum_{0 \leq j \leq n<2 i}\left[\begin{array}{l}
i \\
j
\end{array}\right]_{q^{2}}\left[\begin{array}{l}
i-1 \\
n-j
\end{array}\right]_{q^{2}} q^{2 j} \cdot\left(q^{2 i-1} e^{2 \imath b u}\right)^{n}}\right] .
\end{aligned}
$$

Proof. We can expand the sine and cosine functions by exponentials thus reducing the Fourier series terms in $(14)$ of the form $\cos ((a n+b) u)$ and $\sin ((a n+b) u)$ to $n^{\text {th }}$ powers of the exponential function as

$$
\begin{aligned}
& \cos ((a n+b) u)=\frac{1}{2}\left(e^{(a n+b) \imath u}+e^{-(a n+b) \imath u}\right) \\
& \sin ((a n+b) u)=\frac{1}{2 \imath}\left(e^{(a n+b) \imath u}-e^{-(a n+b) \imath u}\right)
\end{aligned}
$$

Each of these series then follows as a special case of the new infinite series representations proved by Proposition 2.6.

Additional square series expansions related to these forms are derived from the special cases of the Jacobi theta functions defined by $\vartheta_{i}(q) \equiv \vartheta_{i}(0, q)$, and by $\vartheta_{i}^{(j)}(u, q) \equiv \partial^{(j)} \vartheta_{i}\left(u_{0}, q\right) /\left.\partial u_{0}{ }^{(j)}\right|_{u_{0}=u}$ or by $\vartheta_{i}^{(j)}(q) \equiv \vartheta_{i}^{(j)}(0, q)$ for the higher-order $j^{\text {th }}$ derivatives taken over any fixed $j \in \mathbb{Z}^{+}$. For example, we can employ the result for the first-order case in Corollary 2.7 to expand new $q$-series representations for the 
special case series given by

$$
\begin{aligned}
& \vartheta_{1}^{\prime}(q)=2 q^{1 / 4} \times \sum_{n=0}^{\infty}(2 n+1) q^{n^{2}}(-q)^{n} \\
& =2 q^{1 / 4} \cdot \vartheta_{0,1}(2,1 ; q,-q) \\
& (q)_{\infty}^{3}=1-q \times \sum_{n=0}^{\infty}(-1)^{n}(2 n+3) q^{n(n+3) / 2} \\
& =1-q \cdot \vartheta_{0,1}\left(2,3 ; \sqrt{q}, q^{3 / 2}\right) \text {. }
\end{aligned}
$$

We can also form integral representations over the variant forms of the Jacobi theta functions given by $\vartheta_{i}\left(0 \mid \imath x^{2}\right) \equiv \vartheta_{i}\left(e^{-\pi x^{2}}\right)$ as considered in the examples from [7]. For example, when $\Re(s)>2$, we have that $[5, \S 20.10(\mathrm{i})]$

$$
\begin{aligned}
\frac{\left(2^{s}-1\right)}{\sqrt{\pi^{s}}} & \Gamma\left(\frac{s}{2}\right) \zeta(s)=\int_{0}^{\infty} x^{s-1} \vartheta_{2}\left(0, \imath x^{2}\right) d x \\
& =\sum_{i=1}^{\infty} \int_{0}^{\infty}\left[\frac{2 x^{s-1} e^{-\pi x^{2} / 4}(-1)^{i-1} e^{-\pi x^{2}(3 i-4)(i-1)}\left(e^{-2 \pi x^{2}} ; e^{-2 \pi x^{2}}\right)_{i-1} e^{-2 \pi x^{2}(i-1)}}{\sum_{0 \leq j \leq n<2 i}\left[\begin{array}{l}
i \\
j
\end{array}\right]_{e^{-2 \pi x^{2}}\left[\begin{array}{l}
i-1 \\
n-j
\end{array}\right]_{e^{-2 \pi x^{2}}} \times e^{-2 \pi x^{2} \cdot j}\left(-e^{2 \pi x^{2}(i-1)}\right)^{n}}}\right] d x .
\end{aligned}
$$

Remark 3.6 (The General Two-Variable Ramanujan Theta Function). For any non-zero $a, b \in \mathbb{C}$ such that $|a b|<1$, the general two-variable Ramanujan theta function, $f(a, b)$, is expanded by the unilateral series representations

$$
\begin{aligned}
f(a, b) & =1+\sum_{n=1}^{\infty}\left[a^{n(n+1) / 2} b^{n(n-1) / 2}+a^{n(n-1) / 2} b^{n(n+1) / 2}\right] \\
& =1+a \cdot J_{\infty}^{[\mathrm{sq}]}(\sqrt{a b}, a \sqrt{a b})+b \cdot J_{\infty}^{[\mathrm{sq}]}(\sqrt{a b}, b \sqrt{a b}) \\
& =1+\sum_{c \in\{a, b\}} \sum_{i=1}^{\infty} \frac{c \cdot(-1)^{i-1}(a b)^{(3 i-2)(i-1)}(a b ; a b)_{i-1} c^{2 i-2}}{\sum_{0 \leq j \leq n<2 i}\left[\begin{array}{l}
i \\
j
\end{array}\right]_{a b}\left[\begin{array}{l}
i-1 \\
n-j
\end{array}\right]_{a b}(a b)^{j} \cdot\left(-(a b)^{i-1} \cdot c\right)^{n}}
\end{aligned}
$$

which then implies the corresponding new series representations for its special case representations of other special functions. In particular, we have notable special cases of the series in the last equation given by the infinite $q$-Pochhammer product $(q)_{\infty} \equiv f(q):=f\left(-q,-q^{2}\right)$, including an immediate application to expanding new series for the Dedekind eta function, $\eta(\tau)$, and the special cases of Ramanujan's functions, $\varphi(q) \equiv f(q, q)$ and $\psi(q) \equiv f\left(q, q^{3}\right)$.

Explicit special constant values forming real-valued multiples of rational inputs to reciprocals of the gamma function are generated by the expansions of the inputs of $q:=e^{-k \pi}$ to Ramanujan's functions, $\varphi(q) \equiv \vartheta_{3}(q)$ and $\psi(q) \equiv 1 / 2 q^{-1 / 8} \cdot \vartheta_{2}(\sqrt{q})$, for the positive real-valued $k$. The special case series and their corresponding square series integral representations from [7] expanded in (7) of the introduction are only two of a number of special case pairs of these special-function-defined constant forms for the particular real-valued $k \in\{1,2,3,5\} \bigcup\left\{1,2, \frac{1}{2}\right\} \bigcup\{\sqrt{2}, \sqrt{3}, \sqrt{6}\} \bigcup \mathbb{Z}^{+}$cited as examples in the original square series transformations article.

\section{Conclusions}

4.1. Summary. We arrived at formulas for the "square series" subsequences, $c_{h}^{[\mathrm{sq}]}(q)$ and ab ${ }_{h}^{[\mathrm{sq}]}(q)$, implicit to the definition of the infinite J-fraction expansion in (1) and in (2) by computation rather than the combinatorial intuition that motivates many of the J-fraction sequence examples cited in the references $[1,2]$. In Section 2, the J-fraction series expanded by the ratio of the $h^{\text {th }}$ convergent functions, $\mathrm{P}_{h}(q, z)$ and $\mathrm{Q}_{h}(q, z)$, is proved to generate the ordinary geometric square series up to order $h+1$ (or order $2 h$ ) for each finite $h \geq 1$ with the limiting case as $h \rightarrow \infty$ converging exactly to the ordinary square series generating function, $J_{\infty}^{[\mathrm{sq}]}(q, z)$. Moreover, we are able to generate higher-order square series expansions by differentiating the limiting convergent function termwise with respect to $z$. 
We use known properties of the convergent expansions of more general continued fraction representations to prove a new finite sum representation of $\operatorname{Conv}_{h}(q, z)$ given in (9) for each $h \geq 1$. Since the limit of $\operatorname{Conv}_{h}(q, z)$ over $h$ exists as $h$ tends to infinity, these new finite sum results imply new infinite $q$-series representations of both of the ordinary square series cases of $J_{\infty}^{[\mathrm{sq}]}(q, z)$ and $J_{\infty}^{[\mathrm{sq}]}\left(q^{m}, c \cdot q^{p}\right)$ proved in Section 2.3. The latter ordinary square series variant leads to new generating functions for the special function sequences, $r_{p}(n)$ and $\sigma_{1}(n)$, as well as new strictly $q$-series representations of the series for the Jacobi theta functions, particular notable special cases of the Jacobi theta functions, and for the general two-variable Ramanujan theta function given in Section 3.

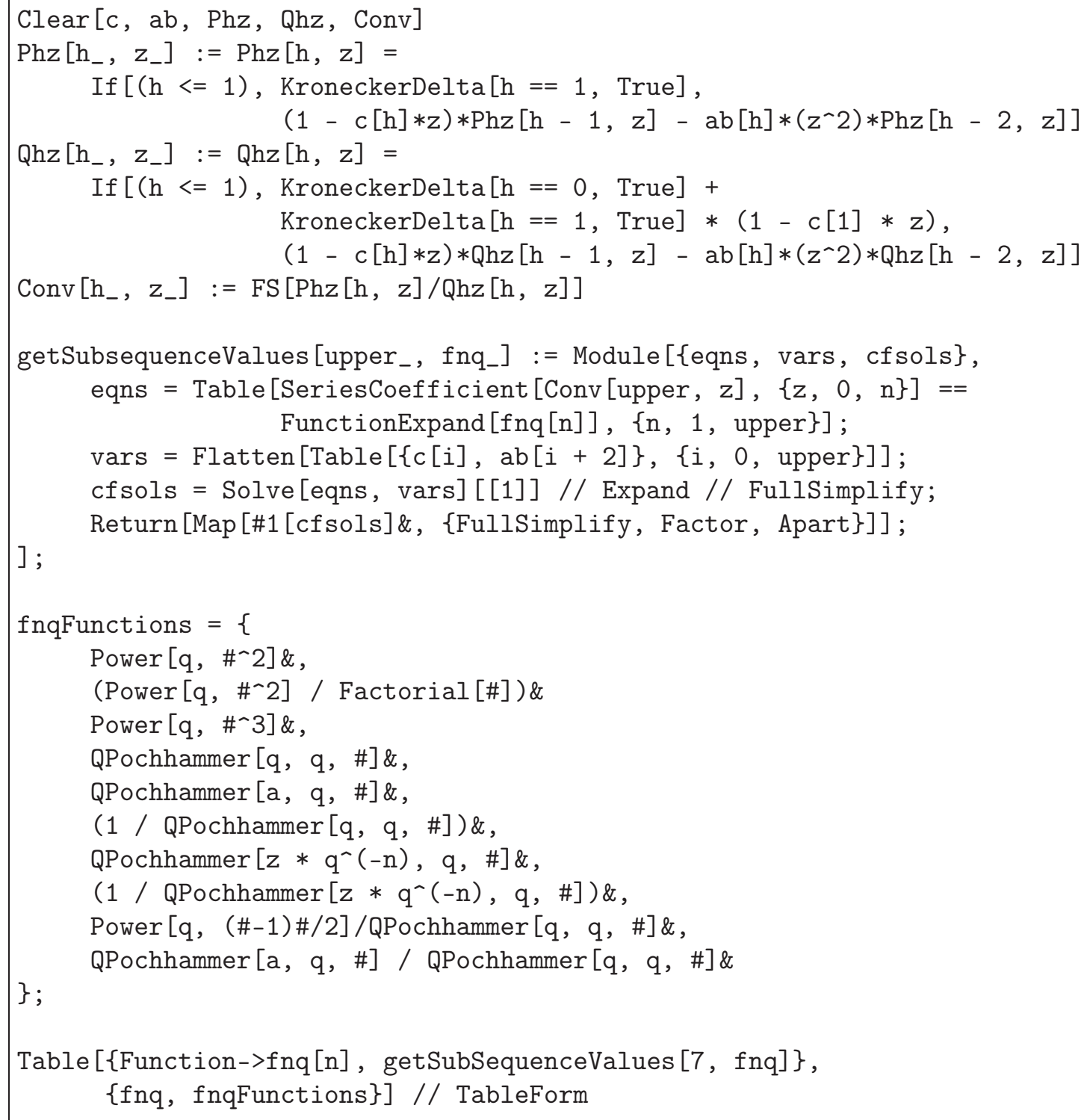

Figure 1. Mathematica Code for Conjecturing New q-Series-Related J-Fraction Expansions

4.2. Remarks on Computationally Conjecturing New J-Fraction Expansions. The listing given in Figure 1 provides a shortened version of the working Mathematica code we used to first computationally conjecture the implicit subsequences involved in our new square series J-fraction expansions in (2). Despite some trial and error with the method illustrated in the figure for conjecturing the new J-fraction expansion results, we do not seem to have correspondingly simple continued fraction representations for the exponential square series case where $f_{n}(q):=q^{n^{2}} / n$ !, which is studied in more detail in the original square series transformations article [7]. The computational methods we used to compute, and subsequently "guess", the 
first few explicit values of the J-fraction component sequences are easily extended, as demonstrated by the example functions in the last table generated in the source code from the figure.

\begin{tabular}{||c||c|l||c|}
\hline$f_{n}(q)$ & $c_{1}(q)$ & $c_{h}(q)(h \geq 2)$ & $\operatorname{ab}_{h}(q)(h \geq 2)$ \\
\hline \hline$(a ; q)_{n}$ & $1-a$ & $q^{h-1}-a q^{h-2}\left(q^{h}+q^{h-1}-1\right)$ & $a q^{2 h-4}\left(a q^{h-2}-1\right)\left(q^{h-1}-1\right)$ \\
$\frac{1}{(q ; q)_{n}}$ & $\frac{1}{1-q}$ & $\frac{q^{h-1}\left(q^{h-1}\left[\begin{array}{c}h-1 \\
1\end{array}\right]_{q}-\left[\begin{array}{c}h-2 \\
1\end{array}\right]_{q}\right)}{\left[\begin{array}{c}2 h-3 \\
1\end{array}\right]_{q}\left(q^{2 h-1}-1\right)}$ & $-\frac{q^{3 h-5}}{\left(q^{2 h-3}-1\right)^{2}\left(1+q^{h-2}+q^{h-1}+q^{2 h-3}\right)}$ \\
$\left(z q^{-n} ; q\right)_{n}$ & $\frac{q-z}{q}$ & $\frac{q^{h}-z-q z+q^{h} z}{q^{2 h-1}}$ & $\frac{\left(q^{h-1}-1\right)\left(q^{h-1}-z\right) \cdot z}{q^{4 h-5}}$ \\
$\frac{1}{\left(z q^{-n} ; q\right)_{n}}$ & $\frac{q}{q-z}$ & $\frac{q^{h-1}\left(q^{2 h-2}+z+q^{h-1} z-q^{h} z\right)}{\left(q^{2 h-3}-z\right)\left(q^{2 h-1}-z\right)}$ & {$\left[\begin{array}{c}h-1 \\
1\end{array}\right]_{q} \cdot \frac{q^{3 h-4}(1-q)\left(q^{h-2}-z\right) \cdot z}{\left(q^{2 h-4}-z\right)\left(q^{2 h-3}-z\right)^{2}\left(q^{2 h-2}-z\right)}$} \\
\hline \hline
\end{tabular}

TABLE 4. Conjectures for Sequence Formulas in $q$-Series-Related J-Fraction Expansions

More precisely, if we let $\left\{f_{n}(q)\right\}_{n=0}^{\infty}$ denote an arbitrary sequence depending on the fixed parameter $q$, we seek to explore the analogous $q$-series-related J-fraction expansions enumerating this sequence in the form of

$$
J_{\infty}(f ; q, z):=\sum_{n=0}^{\infty} f_{n}(q) \cdot z^{n} .
$$

We can always solve for the sequences of implicit coefficients, $\left\{c_{i}\right\}$ and $\left\{\mathrm{ab}_{i}\right\}$, in (1) which (at least formally) generate the sequence of $f_{n}(q)$ over $z$. Typically we find that for more well-known $q$-series generating function forms it is easy to computationally "guess" exact formulas for these sequences, which are each implicit functions of the $f_{i}(q)$ 's, in many special case forms.

Conjectures on the J-Fraction Expansions of Related Series. We conjecture, but do not offer conclusive proofs, that we have related $q$-series generating functions enumerating variants of the sequences, $(a ; q)_{n}$, $(q ; q)_{n}$, and $1 /(q ; q)_{n}$ over $z$ defined by the implicit subsequence listings given in Table 4 . Examples of special series that result by generating the sequences, $f_{n}(q)$, in the table by the properties resulting from our conjectured J-fraction expansions include the following expansions [5, §17] [7, §1.3.5]:

$$
\begin{array}{rlr}
\sum_{n=0}^{\infty}(z ; q)_{n+1} z^{n} & =1+\sum_{n=1}^{\infty}(-1)^{n}\left[q^{n(3 n-1) / 2} z^{3 n-1}+q^{n(3 n+1) / 2} z^{3 n}\right] & \\
e_{q}(z) & =\sum_{n=0}^{\infty} \frac{(1-q)^{n} z^{n}}{(q ; q)_{n}} & \text { (q-Exponential Function) } \\
{ }_{1} \phi_{0}(0 ;-; q, z) & =\sum_{n=0}^{\infty} \frac{z^{n}}{(q ; q)_{n}}=\frac{1}{(z ; q)_{\infty}} & \text { ( } q \text {-Hypergeometric Function) } \\
{ }_{1} \phi_{0}(0 ;-; q, q) & =\sum_{n=0}^{\infty} \frac{q^{n}}{(q ; q)_{n}}=\frac{1}{(q ; q)_{\infty}} & \text { (Partition Function Generating Function) } \\
{ }_{1} \phi_{0}(0 ;-; q, q) & =\left(z q^{-n} ; q\right)_{n} . & \text { (Another } q \text {-Binomial Theorem) }
\end{array}
$$

For the first sequence case in the table, we can extend the methods of proof given in this article for the square series generating functions to find that

$$
\sum_{n=0}^{\infty}(a ; q)_{n} z^{n}=\sum_{i=1}^{\infty} \frac{a^{i-1} q^{(i-1)(i-2)}(a ; q)_{i-1}(q ; q)_{i-1} z^{2 i-2}}{\sum_{0 \leq j \leq n<2 i}\left[\begin{array}{l}
i \\
j
\end{array}\right]_{q}\left[\begin{array}{l}
i-1 \\
n-j
\end{array}\right]_{q}\left(a q^{i-j} ; q\right)_{j}\left(a q^{i-1-n+j} ; q\right)_{n-j} q^{\left(\begin{array}{c}
j \\
2
\end{array}\right)+\left(\begin{array}{c}
n-j \\
2
\end{array}\right)}(-z)^{n}} .
$$

Other examples of new infinite series and q-series expansions for the generating functions of the sequences listed in Table 4, along with many other special cases not considered within the context of the applications cited in this article, are proved similarly. 


\section{REFERENCES}

[1] P. Flajolet, Combinatorial aspects of continued fractions, Discrete Math. 32 (1980), 125-161.

[2] P. Flajolet, On congruences and continued fractions for some classical combinatorial quantities, Discrete Math. 41 (1982), $145-153$.

[3] G. H. Hardy and E. M. Wright, An Introduction to the Theory of Numbers, Oxford University Press, 2008.

[4] S. K. Lando, Lectures on Generating Functions, American Mathematical Society, 2002.

[5] F. W. J. Olver, D. W. Lozier, R. F. Boisvert, and C. W. Clark, NIST Handbook of Mathematical Functions, Cambridge University Press, 2010.

[6] M. D. Schmidt, Jacobi type continued fractions for the ordinary generating functions of generalized factorial functions, J. Integer Seq. 20 (2017).

[7] M. D. Schmidt, Square series generating function transformations, 2016, https://arxiv.org/abs/1609.02803.

[8] H. S. Wall, Analytic Theory of Continued Fractions, Chelsea Publishing Company, 1948.

University of Washington, Department of Mathematics, Padelford Hall, Seattle, WA 98195, USA 